\title{
Fragile Binaries: Observational Leverage on Difficult Astrophysical Problems
}

\author{
T.D. Oswalt ${ }^{1}$, K.B. Johnston ${ }^{1}$, M. Rudkin ${ }^{1}$, \\ T. Vaccaro ${ }^{1}$, and D.Valls-Gabaud ${ }^{2}$ \\ ${ }^{1}$ Dept. of Physics and Astronomy, Florida Institute of Technology \\ 150 West University Blvd., Melbourne, FL 32901 \\ email: toswalt@fit.edu,kyjohnst@fit.edu,mrudkin@fit.edu, tvaccaro@fit.edu \\ ${ }^{2}$ Laboratoire d'Astrophysique UMR CNRS 5572, Observatoire Midi-Pyrénées \\ 14 Avenue Edouard Belin, F-31400 Toulouse Cdx, France. \\ email: David.Valls-Gabaud@obspm.fr
}

\begin{abstract}
Loosely bound, fragile binary stars, whose separations may reach $\sim 0.1 \mathrm{pc}$, are like open clusters with two coeval components. They provide a largely overlooked avenue for the investigation of many astrophysical questions. For example, the orbital distribution of fragile binaries with two long-lived main-sequence components provides a sensitive test of the cumulative effects of the Galactic environment. In pairs where one component is evolved, the orbits have been amplified by post-main-sequence mass loss, potentially providing useful constraints on the initial-to-final mass relation for white dwarfs. The nearly featureless spectra of cool white dwarfs usually provide little information about intrinsic radial velocity, full space motion, population membership, metallicity, etc. However, distant main sequence companions provide benchmarks against which those properties can be determined. In addition, the cooling ages of white dwarf components provide useful limits on the ages of their main sequence companions, independent of other stellar age determination methods. This paper summarizes some of the ways fragile binaries provide useful leverage on these and other problems of interest.
\end{abstract}

Keywords. binaries: common proper motion - stars: white dwarfs

\section{Fragile Binaries: A Definition}

During the first large-scale proper motion surveys of the mid-twentieth century, Luyten (1969 et seq) and Giclas et al. (1971) identified over 6000 very wide common-propermotion binaries $(C P M B s)$ based upon angular proximity in the sky $\left(<300^{\prime \prime}\right)$ and similar proper motion. Most are likely to be low-mass main-sequence pairs (MS+MS). About $10 \%$ of these CPMBs were identified as potentially containing at least one white dwarf (WD) component based upon their location in reduced proper motion diagrams constructed from a color index, apparent magnitudes and proper motions. Luyten chose the symbol $\mathrm{H}(=m+\log \mu+5)$ for reduced proper motion as a tribute to Enjar Hertzsprung, who he credited with the first use of the technique.

The separations of CPMBs range from 10 to $10^{4} \mathrm{AU}$, averaging about $10^{3} \mathrm{AU}$ (Oswalt et al. 1998). CPMBs may not represent the large separation tail of a continuous distribution of binary separations that includes the closer classical visual and spectroscopic binary classes. The latter are believed to form via fragmentation or fission processes; the former may arise from multi-body capture or cluster disintegration processes (see Zinnecker 1984 for an early review). In any case, CPMBs are almost certainly the most common type of binary system (see Luyten 1969). Oswalt \& Smith (1995) and Smith \& Oswalt (1995) have shown that even the current large sample of known CPMBs within $100 \mathrm{pc}$ of the Sun is at most $20 \%$ complete and that many new CPMBs remain to be 
found among the new large-scale surveys such as the Sloan Digital Sky Survey (see Smith et al. 2005) and by combining existing surveys (see Chanamé \& Gould 2004, Lépine \& Shara 2005). We suggest here that the term fragile binary (FB) is more physically descriptive of such loosely bound pairs than the traditional term CPMB, because they are exquisitely sensitive to the influence of the Galactic environment (see below).

\section{Importance of Fragile Binaries with Evolved Components}

FBs provide unique observational leverage on important astrophysical problems that are difficult to address using samples of single stars or clusters. The importance of wide unevolved (MS+MS) pairs is outlined elsewhere in this volume and we defer that discussion to the papers by Allen et al., Chanamé, Sinachopolous et al., Kiyaeva, and Poveda et al.. For the remainder of this paper we will examine only those areas of opportunity afforded by FBs containing at least one evolved component, i.e., WD+MS or WD+WD pairs. FBs with more highly evolved companions (neutron stars or black holes) experienced a supernova explosion that almost certainly disrupted them.

As the end stage of over $90 \%$ of all stars the Galaxy has ever spawned, the observed physical properties of WDs provide important boundary conditions on evolutionary models for stars less massive than $\sim 8 \mathrm{M}_{\odot}$. For example, they constrain the mass-radius relation for degenerate matter and provided one of the first tests of relativity theory, via gravitational redshift measurements. They comprise an accumulating history of the Galaxy containing clues to the initial mass function (IMF), star formation rate (SFR) and initial-to-final mass relation (IFMR) for degenerate stars. Moreover, they provide information about the various components of the Galaxy, e.g., the thick and thin disk, halo and dark matter.

WDs have a very narrow mass distribution with a mean of $\sim 0.6 \pm 0.1 \mathrm{M}_{\odot}$ (Silvestri et al. 2001). Lacking significant energy sources, the evolution of WDs is a simple cooling process in which there is an inverse relation between the age and luminosity. The cooling ages of the oldest WDs played a key role in resolving the discrepancy between the age of the Universe derived from the Hubble constant and globular clusters (Liebert et al. 1988, Oswalt et al. 1996, Bergeron et al. 1997; see also the review paper by Lineweaver 1998).

At present about $500 \mathrm{FBs}$ consisting of WD+MS components have been spectroscopically identified. Most are among the Luyten and Giclas proper motion surveys. Such wide noninteracting pairs are of special interest because the WD component gives information about its MS companion that would otherwise be unobtainable if it were a single star and vice versa. Because a faint star like a WD is many times more likely to be found when it is near a brighter MS star of high proper motion, FBs are likely to harbor a more complete sample of WDs, all other things being equal (search volume, magnitude limit, proper motion limit, etc.). Moreover, in an H-R diagram, the natural dispersion about the WD cooling track is about half the scatter of the MS. Thus, for pairs without trigonometric parallax determinations, a WD component provides a more precise photometric parallax for its MS companion than one based on fits to the MS. Even if trigonometric parallaxes are available, a pair provides two independent distance and luminosity estimates that will be consistent if they are bound. Oswalt et al. (1996) used this leverage to probe a deeper sample of WDs to obtain a new WD luminosity function that set a firm lower limit of $\sim 10$ Gyr to the age of the Galaxy. It also indicated that WDs contribute only a few percent of the dark matter content in the Galaxy.

Among WD+MS pairs, the MS components provide a benchmark for the intrinsic radial velocity and, when trigonometric or photometric parallaxes are available, the full 
space motion of each pair can be computed. With such large orbits, the orbital velocity of both components is $<1 \mathrm{~km} \mathrm{~s}^{-1}$, usually below the precision of measurement in faint stars. Thus, the observed difference in radial velocity between a WD and MS component is essentially the gravitational redshift of the WD. FBs have provided several hundred independent WD mass determinations via gravitational redshift measurements (see Silvestri et al. 2001 and references therein).

Age is one of the most difficult to determine physical properties of single stars. In the years since Skumanich (1972) first introduced it, Barnes (2001), Lachaume et al. (1999), Soderblom et al. (1991) and others have shown that CaII H\&K emission, a proxy for chromospheric activity, provides a reliable age estimate for F, G and K MS stars. For WD components that are warm enough to exhibit Balmer lines, it is relatively easy to estimate cooling ages, temperatures, gravities and final masses from line profile fits, as in Kawka \& Vennes (2006). In a wide non-interacting binary, the cooling age of a white dwarf (WD) component provides a firm lower limit to the age of any distant main sequence (MS) companion. The difference between the apparent ages of the WD+MS components is the time the WD originally spent as a MS star. Using the canonical mass vs. lifetime relation for MS stars (e.g., Cox 2000), one can then estimate the initial mass of the WD, i.e., its mass before post-MS evolution mass loss occurred. The relation between this and a WD's current (i.e., final) mass, the so-called initial-to-final mass relation (IFMR) is one of the weakest links in all of stellar evolution (Jeffries 1997, Weidemann 2000, Catalán 2007). FBs offer a unique opportunity to improve this situation that we are currently exploiting.

Silvestri et al. (2005) attempted to extend the chromospheric activity vs. age relation to $\mathrm{M}$ stars using the ages derived from distant WD companions in 116 FBs. In M stars, H $\alpha$ emission serves as a better proxy for chromospheric activity than Ca II H\&K. The nearby $\mathrm{CaH}_{2}$ and $\mathrm{TiO}_{5}$ bands are useful indicators of effective temperature and metallicity (Reid et al. 1995). Silvestri et al. (2005) found that in general a higher fraction of early $\mathrm{M}$ stars are active (i.e., have strong $\mathrm{H} \alpha$ emission) in accord with studies of $\mathrm{M}$ dwarfs whose ages were derived from cluster membership (Reid et al. 1995). Clearly a different excitation mechanism applies to $\mathrm{M}$ stars than the canonical self-sustaining dynamo in $\mathrm{F}$, $\mathrm{G}$ and $\mathrm{K}$ stars. A much larger sample of $\mathrm{WD}+\mathrm{dM}$ stars will be needed to fully explore where the transition to a proposed 'turbulent dynamo' process occurs, and to identify any additional variables, such as metallicity, that influence whether a star maintains long-lived chromospheric activity or not.

All but a handful of the currently known FBs are members of the Galactic disk (Silvestri et al. 2005, Montiero et al. 2006). Chanamé (2007) is conducting a search of the Sloan Digital Sky Survey to find FBs in the halo. Most will be long-lived low-mass MS+MS pairs whose orbits can set constraints on the dark matter content in the halo (see Chanamé \& Gould 2004). The number of WD+MS pairs found to have large space motions is likely to be sufficient to provide the first robust determination of the WD luminosity function and age determination of the halo. As outlined above, the full space motions of these pairs will be provided by their MS companions regardless of whether their old cool WD companions exhibit measurable lines. Moreover, the metallicities of the WD progenitors is easily measured via their MS companions' spectra and this provides an independent indicator of population membership that single WDs cannot provide.

During post-MS evolution much mass is lost and the orbit of a FB expands. Greenstein (1986) and Oswalt \& Sion (1988) were among the first to present observational evidence for such expansion. Oswalt \& Strunk (1994) and Sterzik \& Durisen (2004) noticed that FBs with MS components of early spectral type tend to have much wider mean separations than those of later spectral class. Valls-Gabaud (1988) and Wood \& 
Oswalt (1998) showed that expansion of up to an order of magnitude can occur with the right combination of initial primary mass and binary mass ratio. Fahiri (2006) showed observational evidence that a gap in the orbital separation distribution may occur near 5 AU. Closer pairs experienced a common envelope phase and their orbits contracted, while wider pairs' orbits expanded as the primary became a WD.

Recently, Johnston et al. (2007) have taken up the challenge of modeling the orbital evolution of FBs in greater detail, including not only the effects of post-MS mass loss, but eventually including the perturbations caused by the Galactic tidal potential, giant molecular cloud encounters, and stellar interactions. Using large samples of FBs drawn from the SDSS, model separation distributions will be constructed for observed samples of evolved (WD+MS, WD+WD) pairs and unevolved (MS+MS) pairs. By matching observed and computed angular orbit separation distributions for Galactic disk FBs, Johnston et al. are attempting to derive average mass loss as a function of average initial mass, thereby achieving a new constraint on the WD IFMR that is independent of other techniques. As the known sample of FBs in the halo becomes large enough, an attempt will be made to model orbit amplification in these ancient pairs as well.

Among the rarest of all FBs are those pairs consisting of two WD components. Luyten (1969) identified about two dozen such pairs in his original proper motion survey. Even today, only about $50 \mathrm{WD}+\mathrm{WD}$ pairs are known. Probably this is because both components tend to be intrinsically faint, but also because the Galaxy is not old enough for many of them to have formed. Sion et al. (1991) showed that WD+WD pairs tend to have slightly smaller average physical separations than WD+MS pairs. This could plausibly have arisen from initial mass ratios that were closer to unity in the progenitors of WD+WD pairs. Because they contain some of the oldest known WDs, such pairs have also played an important role in determining the age of the Galaxy (Oswalt et al. 1996, Bergeron et al. 1997). Holley-Bockelmann examined the possibility that the closest WD+WD pairs will pose a significant source of foreground noise for future gravitational wave detectors.

It came as a surprise to the WD community when Saumon \& Jacobsen (1999) and Hanson (1999) independently predicted that the coolest WDs would not be red in color, but blue, due to the onset of collisionally induced absorption by $\mathrm{H}_{2}$ molecules. It was not long after that the first cool blue degenerate star was actually discovered (Hodgkin et al. 2000). Oppenheimer et al. (2001) stirred up a controversy by finding several dozen such objects in a deep proper motion survey and suggesting that most or all of the dark matter content in the Galactic halo might be cool blue degenerate stars. FBs played an important role in rejecting this conclusion. Silvestri et al. (2002) showed that among $\sim 100$ FBs consisting of WD+MS stars, all but one pair were high velocity members of the thick disk, not the halo. This was a firm conclusion because the MS companions provided radial velocities, gravitational redshift masses, full UVW space motions and metallicities for their WD companions (see Silvestri et al. 2005 for details). Silvestri et al. (2002) showed that the velocity histograms for the WD+MS sample, look like those of Oppenheimer et al. (2001), when degraded by the zero radial velocity assumption necessary for single WDs lacking radial velocity measurements. Recently, Monteiro et al. (2006) and Chanamé (2007) have begun looking for FBs of high space velocity. Such pairs will be very important probes of the age and dark matter content in the halo.

\section{Conclusions}

Much science remains to be gleaned from the many thousands of FBs awaiting study in the large new imaging and proper motion surveys now underway. It is not much of 
a stretch to assert FBs are comparable in importance to open clusters in terms of the leverage they potentially provide on astrophysical problems. Although each has only 2-3 coeval components, FBs span nearly a continuous range of ages over the entire history of the Galaxy, ( 10 Gyr). By comparison only a half dozen or so age milestones are provided by the nearest clusters that contain comparably bright stars. Finally, the shear number of FBs (at least 12,000 WD+MS pairs in the SDSS alone, according to Smith et al. 2005) provide a golden opportunity to attack various problems using subsets that isolate such variables as mass, spectral type, metallicity, age, etc.

Luyten (1969) was right in declaring that we have been ignoring the common man in space by focusing our attention on the more flashy close interacting binaries, OB associations and other rare members of the stellar zoo. He would be pleased to know that several groups are now beginning to use these pairs in ways he could not foresee 40 years ago.

\section{Acknowledgements}

We would like to thank the Scientific Organizing Committee of IAU Symposium 240 for the invitation to present this review and we are grateful to our hosts in Prague for a warm welcome and a productive meeting. One of us (TDO) acknowledges support provided by the U.S. National Science Foundation via grant AST-0206115.

\section{References}

Allen, C., Poveda, A., \& Hemández. Alcántara, A. 2007, these proceedings, 405.

Barnes, S. 2001, "An Assessment of the Rotation Rates of the Host Stars of Extrasolar Planets", ApJ 561, 1095

Bergeron, P., Ruiz, M-T., \& Leggett, S.K. 1997, "The Chemical Evolution of Cool White Dwarfs and the Age of the Local Galactic Disk", ApJS 108, 339

Catalán, S., Ribas, I., Isern, J., García-Berro, E., \& Allende, Prieto, C. 2007, these proceedings, 380.

Chanamé, J. 2007, these proceedings, 316.

Chanamé, J. \& Gould, A. 2004, "Disk and Halo Wide Binaries from the Revised Luyten Catalog: Probes of Star Formation and MACHO Dark Matter", ApJ 601, 289

Cox, A. 2000 (ed.), Allen's Astrophysical Quantities, 4th ed. (New York: AIP Press), Springer

Fahiri, J. 2006, "White Dwarf-Red Dwarf Systems Resolved with the Hubble Space Telescope. I. First Results", ApJ 646, 480

Giclas, H.L., Burnham, R., \& Thomas, N.G. 1971, Lowell Proper Motion Survey: Northern Hemisphere: The G-Numbered Stars. (Flagstaff: Lowell Observatory)

Greenstein, J.L., 1986, "White Dwarfs in Wide Binaries I. Physical Properties", AJ, 92, 859

Hanson, B.M.S. 1999, "Cooling Models for Old White Dwarfs", ApJ 520, 680

Hodgkin, S., Oppenheimer, B., Hambly, N. Jameson, R. Smartt, S., \& Steele, I. 2000, "Infrared Spectrum of an Extremely Cool White Dwarf Star", Nature 403, 57

Jeffries, R.D. 1997, "On the Initial-Final Mass Relation and the Maximum Mass of White Dwarf Progenitors", MNRAS 288, 585

Johnston, K., Oswalt, T., \& Valls-Gabaud, D. 2007 (this volume)

Kawka, A. \& Vennes, S. 2006, "Spectroscopic Identification of Cool White Dwarfs in the Solar Neighborhood", ApJ, 643, 402

Kiyaeva, O. 2007, these proceedings, 131.

Lachaume, R. et al., 1999, "Age Determinations of Main-Sequence Stars: Combining Different Methods", A $\mathscr{G} A 348,897$

Lépine \& Shara, M. 2005, "A Catalog of Northern Stars with Annual Proper Motions Larger than $0^{\prime \prime} .15$ (LSPM-NORTH Catalog)", $A J$ 129, 1483-1522

Liebert, J., Dahn, C., \& Monet, D. 1988, "Luminosity Function of White Dwarfs", ApJ 332, 891 
Lineweaver, C.H. 1999, "A Younger Age for the Universe", Science 284, 1503

Luyten, W.J., 1969, Proper Motion Survey with the Forty-Eight Inch Schmidt Telescope XXI: Double Stars with Common Proper Motion, et seq. (Univ. Minn. Press: Minneapolis)

Monteiro, H., Jao, W-C, Henry, T., Subasavage, J., \& Beaulieu, T. 2006, "Ages of White Dwarf - Red Subdwarf Systems", ApJ 638, 446

Oppenheimer, B. Saumon, D., Hodgkin, S.T., Jameson, R.F., Hambly, N.C., Chabrier, G., Filippenko, A.V., Coil, A.L., \& Brown, M.E. 2001, "Observations of Ultra-Cool White Dwarfs", ApJ 550, 448

Oswalt, T.D., Sion, E.M. 1989, "On the Physical Separations of Wide White Dwarf Binaries", in IAU Coll. 114, White Dwarfs, Springer-Verlag: Berlin, p. 454

Oswalt, T.D. \& Smith, J.A. 1995, "On the Luminosity Function of White Dwarfs in Wide Binaries", in White Dwarfs, eds. D. Koester \& K. Werner, (Springer-Verlag: Heidelberg), p. 113

Oswalt, T., Smith, J., \& Wood, M. 1998, "Wide Binaries: Probes of the Galaxy's Dark Matter Content", Transactions $23^{\text {rd }}$ IAU G/A, Joint Disc. 10, Low Luminosity Stars, (Kluwer: Dordrecht)

Oswalt, T., Smith, J., Wood, M., \& Hintzen, P. 1996, "New Limits to the Galactic Disk Age from the Luminosity Function of White Dwarfs in Wide Binaries", Nature (Letters), 382, 692

Oswalt, T. \& Strunk, D. 1994, "A Catalog of White Dwarfs in Wide Binaries", BAAS 26, 901

Poveda, A., Allen, C., \& Hernández-Aleántara, A., 2007, these proceedings, 417

Reid, I.N., Hawley, S.L., \& Mateo, M. 1995, "Chromospheric and Coronal Activity in Low-Mass Hyades Dwarfs", MNRAS 272, 828

Saumon, D. \& Jacobson, S.B. 1999, "Pure Hydrogen Model Atmospheres for Very Cool White Dwarfs", ApJ 511, L107

Silvestri, N., Oswalt, T., Smith, J.A., Wood, M., Reid, N., \& Sion, E., 2001, "White Dwarfs in Common Proper Motion Binary System: Mass Distribution \& Kinematics", AJ 121, 503

Silvestri, N., Oswalt, T., \& Hawley, S. 2002, "Wide Binary Systems and the Nature of HighVelocity White Dwarfs", AJ 124, 1118

Silvestri, N., Hawley, S., \& Oswalt, T. 2005, "The Chromospheric Activity and Ages of M Dwarf Stars in Wide Binary Systems", ApJ 129, 2428

Sinachopoulos, D., Gauras, P., Medupe, Th., Ducourant, Ch., \& Dionatos, O. 2007, these proceedings, 264

Sion, E. et al. 1991, "The Physical Properties of Double Degenerate Wide Common Proper Motion Systems", AJ 101, 1476

Skumanich, A. 1972, "Time Scales for CA II Emission Decay, Rotational Braking, and Lithium Depletion", ApJ 171, 565

Smith, J.A. \& Oswalt, T. 1995, "Exploration of the Lower Main Sequence Among Wide Binaries", in The Bottom of the Main Sequence and Beyond, ed. C.G. Tinney, (Springer-Verlag: Heidelberg), p. 24

Smith, J.A., Silvestri, N.M., Oswalt, T.D., Harris, H.C., Kleinman, S.J., Munn, J.A., Nitta, A., \& Rudkin, M.A. 2005, "Sloan Digital Sky Survey: Proper Motion Systems Containing White Dwarfs", ASP Conf. 334, 127

Soderblom, D.R., Duncan, D.K., \& Johnson, D.R.H. 1991, "The Chromospheric Emission-Age Relation for Stars of the Lower Main Sequence and Its Implications for the Star Formation Rate", ApJ 375, 722

Sterzik M. \& Durisen, R. 2004, "Are Binary Separations Related to Their System Mass?", in The Environment \& Evolution of Double \& Multiple Stars, Proc. IAU Coll. 191, eds. C. Allen \& C. Scarfe. Rev. Mex. de Astron. y Astrofisica (Conf.) 21, 58

Valls-Gabaud, D. 1988, "Evidence for Mass Loss in Visual Binary Stars", ApESSS 142, 289

Weidemann, V. 2000, "Revision of the Initial-to-Final Mass Relation", A\&A 363, 647

Wood, M. \& Oswalt, T. 1998, "White Dwarf Cosmochronology I. Monte Carlo Simulations of Proper Motion and Magnitude Limited Samples Using Schmidt's $\frac{1}{V_{\max }}$ Estimator", ApJ 497,870

Zinnecker, H. 1984, ApESSS 99, 41 